INPLASY

PROTOCOL

To cite: Cen et al. Clinical efficacy of Asari Radix et Rhizoma preparations for asthma in adults: a protocol for systemic review and metaanalysis. Inplasy protocol

202210023. doi:

10.37766/inplasy2022.1.0023

Received: 06 January 2022

Published: 06 January 2022

Corresponding author:

Mingzheng Cen

cenmingzheng87@163.com

Author Affiliation:

Zhejiang Provincial Hospital of

Chinese Medicine.

Support: $20181228 Y 91$.

Review Stage at time of this submission: The review has not yet started.

Conflicts of interest:

None declared.

\section{Clinical efficacy of Asari Radix et Rhizoma preparations for asthma in adults: a protocol for systemic review and meta-analysis}

Cen, $\mathrm{M}^{1}$; Zhao, $\mathrm{Y}^{2}$.

Review question / Objective: What is the clinical efficacy of Asari Radix et Rhizoma preparations for asthma in adults compared to conventional therapy?

Condition being studied: Adult patients with asthma. There is no restriction regarding the type or severity of asthma.

Information sources: The following electronic database will be searched as information source: Medline, EMBASE database, Cochrane library database, Chinese Biomedicine database (CBM), Chinese National Knowledge Infrastructure (CNKI), Wanfang database, Chongqing VIP database. Manual search will be carried out for any relevant studies, such as references of yielded studies and existing meta-analysis.

INPLASY registration number: This protocol was registered with the International Platform of Registered Systematic Review and Meta-Analysis Protocols (INPLASY) on 06 January 2022 and was last updated on 06 January 2022 (registration number INPLASY202210023).

\section{INTRODUCTION}

Review question / Objective: What is the clinical efficacy of Asari Radix et Rhizoma preparations for asthma in adults compared to conventional therapy?

Condition being studied: Adult patients with asthma. There is no restriction regarding the type or severity of asthma.

\section{METHODS}

Participant or population: Inclusion: Patients with asthma (as diagnosed using any recognized diagnostic criteria). Patients aged above 18. Exclusion: Patients with other respiratory system disease. Pregnant and lactating women. 
Intervention: Patient treated with ARRP alone or combined with other therapy will be included for study. ARRP is defined as any traditional Chinese formula containing ARR, extract of ARR, or active components of ARR. There is no restriction regarding the concentration of ARR substances in the final ARRP. The administered does and frequency of ARRP is not restricted.

Comparator: Participants treated with any recognized treatment other than Asari Radix et Rhizoma preparations. Placebo treatment is also acceptable.

Study designs to be included: Only randomized controlled trials will be included.

Eligibility criteria: 2.1.1 Type of studyOnly RCTs will be included. If literatures haven't claim themselves as RCT clearly but have randomization and comparison, they will also be included.2.1.2 ParticipantsAdult patients with asthma will be included. There is no restriction regarding the diagnostic criteria or type or severity of asthma. Adult is defined as population aged above 18 years. However, asthma patients with other respiratory system disease will be excluded. Pregnant patients and lactating patients will also be excluded.2.1.3 InterventionsPatient treated with ARRP alone or combined with other therapy will be included for study. ARRP is defined as any traditional Chinese formula containing ARR, extract of ARR, or active components of ARR. There is no restriction regarding the concentration of ARR substances in the final ARRP. The administered does and frequency of ARRP is not restricted.2.1.4 ComparisonPatients received any pharmacological treatments other than ARRP will be included. Placebo treatment is also acceptable.

Information sources: The following electronic database will be searched as information source: Medline, EMBASE database, Cochrane library database, Chinese Biomedicine database (CBM), Chinese National Knowledge Infrastructure (CNKI), Wanfang database, Chongqing VIP database. Manual search will be carried out for any relevant studies, such as references of yielded studies and existing metaanalysis.

Main outcome(s): The primary outcome is clinical effective rate. Effective is defined as disappear or relief of asthma symptoms.

Quality assessment / Risk of bias analysis: The quality of eligible studies will be assessed using the Cochrane tool for risk of bias.

Strategy of data synthesis: The extracted data will be going through before final data synthesis. If eligible studies reporting primary outcomes are less than 5, only descriptive presentation will be carried out. Otherwise, Review Manager software (Version 5.3, Copenhagen, The Nordic Cochrane Center, The Cochrane collaboration, 2014) is applied for the data synthesis. Heterogeneity among studies will be assessed using 12 test before synthesis. Mantel-Haenszel fixed effect model will be used for data synthesis if 12 is no more than $50 \%$, or random effect model will be used because heterogeneity exists among studies. Odds ratio (OR) and 95\% confidential interval $(\mathrm{Cl})$ will be calculated for categorical results, and mean difference (MD) and $95 \% \mathrm{Cl}$ will be calculated for continuous outcomes.

Subgroup analysis: (1) combined therapies; (2) specific formulation of ARR; (3) severity and type of asthma.

Sensitivity analysis: Sensitivity analysis will be carried out using the leave-one-out method.

Country(ies) involved: China.

Keywords: Asari Radix et Rhizoma; asthma; complementary medicine; traditional Chinese Medicine; efficacy.

Contributions of each author:

Author 1 - Mingzheng Cen.

Email: cenmingzheng87@163.com

Author 2 - Yuhua Zhao.

Email: zhaoyuhua1987@126.com 